\title{
ENSEÑANZAY APRENDIZAJE DE LAS CIENCIAS NATURALES. UN ANÁLISIS DEL CONTEXTO DE EDUCACIÓN BÁSICA PRIMARIA
}

\section{TEACHING AND LEARNING OF NATURAL SCIENCES. AN ANALYSIS OF THE CONTEXT OF BASIC PRIMARY EDUCATION}

\section{Carlos Antonio Pabón Galán ${ }^{1}$}

UFPS

\section{RESUMEN}

El artículo tiene como objetivo describir el proceso de enseñanza y aprendizaje de las ciencias naturales en el nivel de básica primaria desde las opiniones de docentes y estudiantes. Se enmarcó en un enfoque mixto de tipo cuantitativo y cualitativo. Para recopilar datos cuantitativos, se utilizó un proceso de muestreo para seleccionar 15 docentes que enseñan ciencias naturales en el nivel de educación básica primaria y 60 estudiantes de 5 to grado. Se concluyó que la práctica pedagógica en el área de ciencias naturales se centra en el docente con un estudiante poco activo y limitado para actividades prácticas y de discusión. Las barreras que enfrentan los docentes para

$1 \quad$ Magister en Ciencias e Ingeniería de los Alimentos - Universidad Politécnica de Valencia (España). Docente investigador de la Universidad Francisco de Paula Santander (Colombia). Correo electrónico: carlosantoniopg@ufps. edu.co. Orcid: 0000-0003-4027-819X. enseñar ciencias naturales están relacionadas con aulas con alto número de estudiantes, recursos y materiales insuficientes, falta de preparación y competencias del docente a nivel disciplinar y pedagógica, así como ausencia de proceso de evaluación del aprendizaje.

PALABRAS CLAVE: Práctica pedagógica, enseñanza, aprendizaje, educación básica, evaluación, estrategias

\section{SUMMARY}

The aim of this article is to describe the teaching and learning process of natural sciences at primary school level from the point of view of teachers and students. It was framed in a mixed quantitative and qualitative approach. To collect quantitative data, a sampling process was used to select 15 teachers who teach natural sciences at primary school level and 60 th grade 
students. It was concluded that pedagogical practice in natural sciences is teacher-centred with little active student involvement and limited hands-on activities and discussion. The barriers faced by teachers in teaching natural sciences are related to classrooms with high numbers of students, insufficient resources and materials, lack of teacher preparation and competences at the disciplinary and pedagogical levels, as well as the absence of a learning assessment process.

KEYWORDS: Pedagogical practice, teaching, learning, basic education, assessment, strategies.

\section{INTRODUCCIÓN}

La educación es necesaria para el crecimiento y desarrollo de una sociedad. En Colombia, para asegurar una educación de calidad, el sistema educativo se divide en los siguientes niveles educativos: preescolar, básica que incluye la primaria y secundaria, media y superior. Entre estas etapas, la educación básica primaria merece una atención especial debido a que es la base para los niveles siguientes.

A lo largo de las décadas, se han logrado avances significativos en la educación básica primaria en Colombia, especialmente en cuanto acceso y cobertura. Lamentablemente, el rendimiento de los estudiantes de este nivel fue comparativamente menor de lo esperado (Aguilar-Barreto, et al 2017; Montes, 2017; Hernández-Suárez et al., 2021; Hernández et al., 2021). Montes (2017) describió la calidad de la educación básica primaria en Colombia como deficiente, lo que lleva a los resultados poco alentadores. Esta situación se apoya aún más en el Informe de Organización para la Cooperación y el Desarrollo Económicos (OCDE, 2016), hace referencia a que "las tres asignaturas evaluadas por PISA son: Lectura, matemáticas y ciencias en 15 países, más de uno de cada dos estudiantes tienen un bajo rendimiento en matemáticas" (p.34).

Del resultado del informe PISA 2018, prueba que evaluó a los estudiantes de 15 años para medir qué tan preparados están para entender y enfrentar los retos prácticos de la vida adulta, muestra un panorama educativo poco alentador para Colombia, a pesar del crecimiento que se ha tenido desde el 2006, donde se concluye que los estudiantes en matemáticas obtuvieron un rendimiento menor (391) que la media de la OCDE (481), de los cuales casi $40 \%$ tuvieron un bajo nivel de logro (Echazarra \& Schwabe, 2019).

Los resultados del nivel de competencia de los estudiantes evidencian la prestación de una educación de mala calidad (Prada et al., 2018). Este argumento también es respaldado por Rincón et al. (2017), de que la calidad del aprendizaje y la enseñanza de la lectura, matemáticas y ciencias naturales en las escuelas del país distan mucho de ser satisfactoria. De hecho, la mayoría de las escuelas tienden a embarcarse en la memorización y enseñanza tradicional y hacen el mínimo esfuerzo en desarrollar competencias y habilidades (Salamanca \& Hernández, 2018). Por lo tanto, la calidad de las disciplinas escolares, especialmente las ciencias naturales, es un tema importante que debe abordarse con urgencia.

Los docentes pueden lograr una educación de calidad, si desarrollan una variedad de técnicas de aprendizaje y enseñanza innovadoras que faciliten un entorno de aprendizaje de apoyo para el desarrollo de las competencias en sus estudiantes (Veloza \& Hernández, 2018a, 2018b; Hernández \& Salamanca, 2018). El docente es un elemento orientador importante en el aprendizaje de los estudiantes (HernándezSuárez et al., 2017). 
Mora y Hernández (2017), proporcionan evidencia que respalda que las intervenciones innovadoras dirigidas al aprendizaje de los estudiantes funcionaron a través de los docentes. De manera similar, Albarracín et al. (2020), también aportan evidencia de que los docentes eficaces e innovadores tienen más probabilidades de demostrar una gestión proactiva de las lecciones, bien organizadas con objetivos claros, y un entorno que apoyó su labor.

Sin embargo, en algunos docentes siguen manejando prácticas tradicionales a pesar de la implementación de políticas educativas, que buscan su mejoramiento continuo (Hernández et al., 2021; Hernández-Suárez et al., 2021). Por lo tanto, los cambios que el docente puede implementar para mejorar el aprendizaje pueden variar en función de numerosos factores y necesitan continuamente estar evaluados para mejorar su desempeño y práctica pedagógica (Hernández et al., 2020). La formación y el desarrollo profesional docente son importantes para preparar docentes de mejor calidad, que sean capaces de demostrar sus competencias.

En Colombia, el Ministerio de Educación Nacional - Mineducación, plantea como objetivo principal el mejoramiento de la calidad educativa en todos los niveles, desde la primera infancia hasta la superior. $Y$ en aras de lograr esta meta, se diseñó Todos a Aprender: el Programa de Transformación de la Calidad Educativa, mejor conocido como PTA (Proyecto Todos a Aprender) cuyo propósito es mejorar los aprendizajes de los estudiantes de básica primaria en lenguaje y matemáticas del país, de establecimientos educativos que muestran desempeño insuficiente (Mineducación, 2014).

El PTA plantea la puesta en marcha de acciones pedagógicas encaminadas a fortalecer las prácticas en el aula, brindar referentes curriculares claros que indiquen los objetivos de aprendizaje, desarrollar herramientas apropiadas para la evaluación y trabajar en la selección y uso de materiales educativos para los docentes y estudiantes, los cuales deben estar acordes con los ambientes de aprendizajes. Asimismo, se definió un plan de formación y acompañamiento para los docentes en sus propias aulas (formación situada), ya que es en la interacción entre pares y docentes con sus estudiantes donde ocurren las verdaderas transformaciones educativas (Mineducación, 2014).

Por lo tanto, cualquier iniciativa de mejora de la calidad de la educación en ciencias naturales en el nivel de básica primaria en Colombia necesita una mejor comprensión del proceso de enseñanza aprendizaje. Lamentablemente, hasta ahora hay una investigación limitada sobre las prácticas pedagógicas en ciencias naturales en las escuelas primarias que son beneficiarias del programa PTA. Por tanto, es útil un estudio relacionado con el proceso de enseñanza y aprendizaje en el aula en la enseñanza de las ciencias naturales.

El objetivo de este estudio es examinar el proceso de enseñanza y aprendizaje de las ciencias naturales, específicamente la práctica pedagógica de los docentes, los métodos y técnicas, las prácticas de evaluación para el aprendizaje y la participación de los estudiantes de básica primaria.

\section{MARCO CONCEPTUAL}

El enfoque constructivista se ha centrado en el proceso de adquirir conocimiento científico (basado en la investigación) y en alentar a los estudiantes a comprender la naturaleza de la investigación científica en lugar del conocimiento enseñado (Salamanca \& Hernández, 2018). El constructivismo que surgió de los trabajos de Piaget, Vygotsky y Ausubel (Ortiz, 2015). Palma (2017), señala que el uso de principios didácticos constructivistas ayuda a los docentes de primaria a mejorar sus técnicas 
de aprendizaje y enseñanza que permiten a los estudiantes desarrollar sus habilidades de pensamiento y ampliar su experiencia de aprendizaje (Fernández-Cezar et al., 2019).

Durante los últimos años, existe una creciente demanda de desarrollo profesional docente en el área de ciencias naturales en todos los niveles educativos incluidos el nivel de básica primaria (Gamboa et al., 2020). Varios estudios reconocieron el valor del desarrollo profesional docente que incluye un enfoque de aprendizaje orientado a la indagación seguido del uso de un modelo de aprendizaje constructivista, por ejemplo, actividades científicas prácticas, métodos de enseñanza basados en la investigación, aprendizaje cooperativo, entre otros (Salamanca \& Hernández, 2018; Hernández \& Salamanca, 2018). HernándezSuárez, et al. (2017) argumentan que el desarrollo profesional de los docentes debería centrarse en desarrollar la competencia de los docentes para que puedan adaptar mejor su enseñanza al contexto en lugar de los métodos establecidos para la enseñanza.

\section{MÉTODO}

\section{DISEÑO DE LA INVESTIGACIÓN}

Para el propósito de este estudio, se utilizó un método cuantitativo. Por lo tanto, este estudio examinó el proceso de enseñanza y aprendizaje de las ciencias naturales a través de encuestas. $\mathrm{El}$ enfoque cuantitativo incluye cuestionarios tanto para docentes como para estudiantes.

\section{POBLACIÓN Y MUESTRA}

El estudio se realizó en la secretaria de educación del municipio de Cúcuta, Colombia. El estudio comprendió como población objeto de estudio dos instituciones educativas que son beneficiarias del PTA. Se seleccionaron 15 docentes que orientan ciencias naturales y 60 estudiantes de 5to grado, que son los estudiantes por edad que más desarrollo cognitivo tienen en comparación con los grados inferiores, de los cuales el $50 \%$ eran estudiantes y el $50 \%$ eran niñas para asegurar el equilibrio de género. Se encuestó a 40 docentes de ciencias naturales y de ellos, 15 completaron y devolvieron cuestionarios con una tasa de respuesta del $37,5 \%$. La muestra de docentes quedo compuesta por el $32 \%$ para el género masculino y el $68 \%$ para el femenino.

\section{INSTRUMENTOS}

Este estudio se llevó a cabo utilizando la técnica de encuesta mediante cuestionarios, uno para docentes y otro para estudiantes. La razón para elegir los cuestionarios es que se pueden utilizar para recopilar una gran cantidad de información en un tiempo limitado y los resultados de los cuestionarios se pueden analizar de manera más metódica y decidida en comparación con los otros tipos de instrumentos de investigación (Casas et al., 2003). Se utilizó un formato de respuesta de cuatro puntos (totalmente de acuerdo, de acuerdo, en desacuerdo y totalmente en desacuerdo) para ambos cuestionarios con el fin de lograr resultados más sencillos.

El cuestionario para docentes se dividió en 5 secciones, tales como información socioacadémica, las opiniones sobre el propósito de la enseñanza, proceso de enseñanza y aprendizaje en el aula, factores que impiden mejorar la calidad de la enseñanza y recomendaciones para mejorar la calidad de la enseñanza de las ciencias naturales. El cuestionario para los estudiantes constaba de tres secciones, a saber, información demográfica, opiniones de los estudiantes sobre la práctica real en el aula y preguntas relacionadas con el propósito de su aprendizaje y cómo se puede mejorar su aprendizaje en ciencias naturales.

Los cuestionarios de este estudio fueron evaluados por tres especialistas en el campo de la enseñanza de las ciencias para asegurar la validez del contenido. Sus sugerencias fueron 
consideradas e incorporadas. Los cuestionarios se sometieron a pruebas piloto seleccionando al azar docentes y estudiantes. El resultado del piloto se utilizó para mejorar aún más las preguntas de los cuestionarios.

\section{PROCEDIMIENTO}

Se utilizó el software estadístico SPSS V. 24 para analizar los ítems de las escalas en los cuestionarios de los docentes y estudiantes. Las opiniones de los encuestados se codificaron en grupos y se calculó la frecuencia de las respuestas de cada grupo. Se utilizó un enfoque estadístico descriptivo para presentar los datos numéricos utilizando porcentajes.

\section{RESULTADOS Y DISCUSIÓN \\ INFORMACIÓN DEL CONTEXTO}

Los datos revelaron que casi la mitad de los docentes (46\%) tenían una maestría y una gran mayoría de los docentes incluidos en la muestra (83\%) eran Licenciados en Ciencias Naturales, Biología y Química, Matemáticas y Física y otras áreas. Más de la mitad de los docentes $(53 \%)$ tenían entre 6 y 20 años de experiencia en la enseñanza. Casi todos los docentes enseñan ciencias naturales junto con otras materias y muy pocos docentes $(3 \%)$ solo enseñaban ciencias naturales. El tamaño de las clases oscilaba entre 10 y 50 estudiantes y el tamaño de clase más común era entre 40 y 45 estudiantes. El número promedio de estudiantes en una clase de ciencias naturales fue de 36.6.

\section{FUNDAMENTACIÓN DE LA ENSEÑANZA Y EL APRENDIZAJE DE LAS CIENCIAS NATURALES}

Tabla 1. Objetivos de la enseñanza y el aprendizaje de las ciencias

\begin{tabular}{|l|c|c|}
\hline Objetivos de la enseñanza y el aprendizaje de las ciencias & $\mathrm{N}$ & $\%$ \\
Docentes & 4 & 26.7 \\
\hline Ayudar a los estudiantes a leer y comprender el contenido. & 4 & 26.7 \\
Motivar a los estudiantes a adquirir los conocimientos de la ciencia & 7 & 46.7 \\
Apoyar a los estudiantes para que desarrollen comprensión científica & 2 & 13.3 \\
Preparar a los estudiantes para la siguiente clase de ciencias & 2 & 13.3 \\
Aprobar los exámenes & 3 & 20.0 \\
Ayudar a los estudiantes a comprender el contexto del mundo moderno & 1 & 7.0 \\
Permitir que los estudiantes apliquen la ciencia en su vida cotidiana & 29 & 48.3 \\
\hline Estudiantes & 25 & 41.6 \\
\hline Aprobar el examen & 14 & 23.3 \\
Aprobar el examen con una puntuación alta & 18 & 30.0 \\
Convertirse en un profesional de las ciencias & 8 & 13.3 \\
\hline Saber acerca del conocimiento científico
\end{tabular}

Nota: Algunos encuestados respondieron más de una respuesta, por lo que el porcentaje total es superior a 100 .

Los datos de la Tabla 1 revelan que, aproximadamente la mitad de los docentes (46\%) creen que el objetivo principal de la enseñanza de la ciencia es desarrollar la comprensión científica y desarrollar la alfabetización científica. También el 20\% afirmó que era 
importante lograr que los estudiantes aplicaran y comprendieran el contexto más amplio de la ciencia en su vida cotidiana. Sin embargo, alrededor del $13 \%$ de los docentes afirmó que la principal preocupación era la preparación de exámenes y la preparación de los estudiantes para la siguiente fase de su aprendizaje. La mayoría de los estudiantes señaló que aprenden ciencias naturales para aprobar los exámenes, sin embargo, un número menor de estudiantes (30\%) aprende ciencias naturales para mejorar sus conocimientos científicos.

Aproximadamente la mitad de los docentes cree que el propósito de enseñar ciencias naturales en el nivel primario es desarrollar la capacidad científica e iniciar la investigación de los estudiantes. Estos puntos de vista están en consonancia con los lineamientos curriculares del Ministerio de Educación Nacional (1998, 2004, 2016) que establecen que uno de los objetivos de la educación en ciencias es preparar a los estudiantes para desarrollar sus competencias, conocimientos, habilidades y actitudes científicas. Estos hallazgos están respaldados por Veloza \& Hernández, (2018a, 2018b), Hernández \& Salamanca (2018) y Salamanca \& Hernández (2018) que en sus investigaciones evidencia que la enseñanza de las ciencias en los niveles básico ayudar al estudiante a desarrollar sus habilidades de pensamiento cognitivo y comprender el contexto a través de la investigación.

\section{PROCESO DE ENSEÑANZA Y APRENDIZAJE}

Tabla 2. Opiniones de los docentes y de los estudiantes sobre el alcance del proceso de enseñanza y aprendizaje

\begin{tabular}{|c|c|c|}
\hline \multicolumn{3}{|l|}{ Alcance del proceso de enseñanza y aprendizaje } \\
\hline & $\mathrm{TA}+\mathrm{DA}$ & $E D+T D$ \\
\hline \multicolumn{3}{|l|}{ Docentes } \\
\hline $\begin{array}{l}\text { Al final de las actividades se produce una discusión en toda la } \\
\text { clase con resúmenes de las ideas principales }\end{array}$ & 73.3 & 26.7 \\
\hline $\begin{array}{l}\text { Se anima a los estudiantes a planificar sus propios experimentos } \\
\text { para investigar sus propias preguntas }\end{array}$ & 20.0 & 80.0 \\
\hline $\begin{array}{l}\text { Utilizo una serie de estrategias flexibles para satisfacer las } \\
\text { necesidades de los estudiantes }\end{array}$ & 60.0 & 40.0 \\
\hline Los estudiantes están muy comprometidos con mi clase. & 66.7 & 33.3 \\
\hline $\begin{array}{l}\text { Para compartir y mejorar las estrategias de enseñanza, los } \\
\text { docentes de ciencias de este centro observan regularmente las } \\
\text { clases de los demás }\end{array}$ & 53.3 & 46.7 \\
\hline \multicolumn{3}{|l|}{ Estudiantes } \\
\hline El docente hace preguntas, pero él mismo las responde. & 51.7 & 48.3 \\
\hline Observo al docente hacer experimentos & 68.3 & 31.7 \\
\hline Escucho al docente explicando su clase & 93.3 & 6.7 \\
\hline Sé qué hacer y a quién preguntar si me cuesta trabajo & 40.0 & 60.0 \\
\hline Escribo mucho en la clase de ciencias & 41.7 & 58.3 \\
\hline Puedo explicar mis ideas a otras personas & 40.6 & 59.4 \\
\hline
\end{tabular}


Los datos de la Tabla 2 indican que el $73 \%$ de los docentes realizaron un discusión de toda la clase con resúmenes de las ideas principales al final de las actividades diarias de la clase. El $60 \%$ docentes se empeña en utilizar una amplia serie de estrategias flexibles para satisfacer las necesidades de los estudiantes. Una pequeña parte de los docentes (20\%) anima a los estudiantes a planificar sus propios experimentos para investigar sus propias preguntas. Algo, más de la mitad de los docentes $(53 \%)$ no observan las clases de los demás como parte de compartir y mejorar las estrategias de enseñanza y aproximadamente un tercio cree que los estudiantes no están muy comprometidos en sus clases.
Los datos de la Tabla 2 muestran que casi todos los estudiantes (93\%) escuchan la explicación del docente toda o la mayor parte de la clase. Sin embargo, la mayoría de los estudiantes $(59 \%)$ informó que existe un margen limitado para explicar sus ideas a los demás. Los estudiantes escuchan y ven con frecuencia las demostraciones de los docentes. Desilusionadamente, cuando un docente hace preguntas, aproximadamente la mitad de los estudiantes afirman que los docentes también brindan las respuestas. Un número considerable de estudiantes informó que completan mucho la escritura en el aula (42\%) y no saben qué hacer ni a quién preguntar si encuentran trabajo duro $(40 \%)$.

Tabla 3. Comparación de las opiniones de los docentes y estudiantes sobre el proceso de enseñanza y aprendizaje.

\begin{tabular}{|l|c|c|}
\hline & Estudiantes & Docentes \\
\hline Solución de problemas & 25.0 & 60.6 \\
\hline Preguntas de los estudiantes & 36.9 & 58.7 \\
\hline Trabajos prácticos & 34.4 & 63.3 \\
\hline Preguntas sobre el aprendizaje de los estudiantes & 29.3 & 53.4 \\
\hline Los estudiantes hablan & 32.0 & 86.0 \\
\hline Lectura del libro de texto & 93.3 & 74.7 \\
\hline Trabajo en grupo & 56.7 & 80.0 \\
\hline Comprobación del libro de texto & 77.0 & 88.6 \\
\hline
\end{tabular}

Una comparación de las percepciones de los estudiantes y los docentes, en la Tabla 3 reveló que solo alrededor de un tercio de los estudiantes hacen preguntas directas $(37 \%)$ y un número similar tiene la oportunidad de realizar trabajos prácticos y charlas en el aula. Un número menor de estudiantes (25\%) comienzan sus lecciones con preguntas o plantean problemas y hacen preguntas sobre el aprendizaje. Estos resultados contrastan fuertemente con las opiniones de los docentes, donde la mayoría de ellos afirman que sus lecciones comienzan con el planteamiento de problemas y los estudiantes tienen la oportunidad de hablar y se les proporcionan actividades prácticas. Ambos grupos están de acuerdo en que leer libros de texto y revisar el cuadernos es el método principal de entrega de información en la mayoría de las clases. Sin embargo, Tapia et. al. (2011) argumentaron que escuchar al estudiante y hacer preguntas sobre cómo aprenden, qué están aprendiendo y cómo saben que lo han aprendido, es importante para mejorar el aprendizaje y el rendimiento. Además, (Zona-López \& Giraldo-Márquez, 2017) hacen hincapié en iniciar las clases planteando un problema e invitar a los estudiantes para resolverlos y desarrollar sus habilidades de pensamiento. 


\section{EVALUACIÓN}

Tabla 4. Respuestas de los docentes sobre la evaluación

\begin{tabular}{|l|c|c|}
\hline Evaluación & TA + DA & ED + TD \\
\hline $\begin{array}{l}\text { Doy retroalimentación oral y califico a los estudiantes para que } \\
\text { sepan lo bien que lo están haciendo y lo que tienen que hacer } \\
\text { para mejorar }\end{array}$ & 93.3 & 6.7 \\
\hline $\begin{array}{l}\text { Analizo los resultados de la evaluación para identificar las } \\
\text { diferencias en el rendimiento de los estudiantes. }\end{array}$ & 80.0 & 20.0 \\
\hline Utilizo la información de la evaluación para la planificación futura. & 53.3 & 46.7 \\
\hline $\begin{array}{l}\text { Evalúo los conocimientos de los estudiantes y los utilizo como } \\
\text { guía para la planificación de las clases. }\end{array}$ & 53.3 & 46.7 \\
\hline $\begin{array}{l}\text { Evalúo los resultados de los estudiantes revisando su cuaderno o } \\
\text { sus exámenes escritos. }\end{array}$ & 88.6 & 11.3 \\
\hline $\begin{array}{l}\text { Nota: TA = Totalmente de acuerdo; DA = De acuerdo; ED = En } \\
\text { desacuerdo; TD = Totalmente en desacuerdo. }\end{array}$ & \\
\hline
\end{tabular}

En la Tabla 4, se puede ver que la gran mayoría de los encuestados (89\%) afirmó que las estrategias de evaluación más utilizadas son revisar los cuadernos o los exámenes escritos, así como él 93\% de los docentes proporcionan retroalimentación oral. Aproximadamente un poco más de la mitad de los docentes (53\%) están de acuerdo en que no utilizaron la información de la evaluación para la planificación futura y casi la mitad de los docentes no evaluó el conocimiento existente de los estudiantes. Estos resultados son consistentes con Vargas (2014) que indicó que los docentes se enfocan principalmente en la evaluación escrita.

\section{RECURSOS E INFRAESTRUCTURA PARA EL PROCESO DE ENSEÑANZA Y APRENDIZAJE.}

Tabla 5. Recursos e infraestructura disponible para los docentes de ciencias naturales.

Mejoras en los recursos e infraestructura en los últimos cinco años Suficiente espacio en mi aula según el número de estudiantes y las actividades ofrecidas

Tengo la oportunidad de utilizar materiales TIC

La escuela proporciona todos los materiales

Suficientes materiales de ciencias para utilizar en mis clases

\begin{tabular}{c|c} 
No & Si \\
20.0 & 80.0 \\
60.0 & 40.0 \\
& \\
73.3 & 26.7 \\
73.3 & 26.7 \\
66.7 & 33.3
\end{tabular}

(67\%). Además, alrededor del 73\% informaron que sus escuelas no proporcionan materiales suficientes y tienen muy pocas oportunidades de utilizar estrategias digitales. Sin embargo, la
Como se presenta en la Tabla 5, la mayoría de los docentes incluidos en la muestra indicaron que carecen de espacio en el aula (60\%) y tienen materiales didácticos insuficientes 
gran mayoría está de acuerdo en que se han realizado mejoras durante los últimos cinco años en el suministro de recursos.

Tabla 6. Recursos e infraestructura.

\begin{tabular}{|l|c|c|c|}
\hline Recursos e infraestructura & Bueno & Satisfactorio & Malo \\
\hline Calidad de los libros o textos & 40.0 & 53.3 & 6.7 \\
\hline Calidad de las guías de enseñanza & 26.7 & 53.3 & 20.0 \\
\hline Apoyo de los jefes académicos & 13.3 & 40.0 & 46.7 \\
\hline
\end{tabular}

Los datos de la Tabla 6 muestran que casi todos los docentes $(93 \%)$ informaron que la calidad de los libros de texto de ciencias naturales de los estudiantes es buena o satisfactoria. Lo que evidencia que los libros de ciencias juegan un papel importante en la enseñanza y como los docentes los usan en clase, más que las guías, es el libro el que dicta el programa (Caldeira, 2005). Por otro lado, de los encuestados se infiere que los docentes no reciben el apoyo suficiente de sus jefes académicos.

\section{CONOCIMIENTO Y PROCESO DE ENSEÑANZA DE LOS DOCENTES}

Tabla 7. Opiniones de los docentes sobre el conocimiento y el proceso de enseñanza de las ciencias.

\begin{tabular}{|l|c|c|}
\hline \multicolumn{1}{|c|}{ Opiniones } & TA + DA & ED + TD \\
\hline $\begin{array}{l}\text { Puedo demostrar un buen conocimiento actualizado de la materia } \\
\text { en todos los temas. }\end{array}$ & 53.3 & 46.7 \\
\hline Tengo grandes expectativas para todos los estudiantes & 80.0 & 20.0 \\
\hline $\begin{array}{l}\text { Creo que la discusión en grupos en la clase es útil para el } \\
\text { aprendizaje de los estudiantes. }\end{array}$ & 86.7 & 13.3 \\
\hline $\begin{array}{l}\text { Creo que es importante dedicar tiempo a la preparación completa } \\
\text { antes de impartir la clase }\end{array}$ & 93.3 & 6.7 \\
\hline $\begin{array}{l}\text { Sé utilizar estrategias de enseñanza orientadas a la indagación/ } \\
\text { investigación }\end{array}$ & 53.3 & 46.7 \\
\hline Estoy satisfecho con la calidad de la enseñanza en mis clases & 60.0 & 40.0 \\
\hline Comparto mis objetivos de aprendizaje con los estudiantes & 46.7 & 53.3 \\
\hline Me tomo la mayor parte del tiempo para presentar los contenidos & 66.7 & 33.3 \\
\hline $\begin{array}{l}\text { Tengo tiempo durante la semana para trabajar con mis compañeros } \\
\text { en el plan de estudios y la enseñanza de las ciencias }\end{array}$ & 46.6 & 53.3 \\
\hline $\begin{array}{l}\text { Tengo suficientes oportunidades para asistir a la formación y a los } \\
\text { talleres para mejorar mi enseñanza }\end{array}$ & 33.3 & 66.7 \\
\cline { 2 - 3 } $\begin{array}{l}\text { Nota: TA = Totalmente de acuerdo; DA = De acuerdo; ED = En } \\
\text { desacuerdo; TD = Totalmente en desacuerdo. }\end{array}$ & & \\
\cline { 2 - 3 } & &
\end{tabular}


Los datos de la Tabla 7 revelan que la gran mayoría de los docentes $(80 \%)$ tenían altas expectativas de todos los estudiantes. También creen que tomarse un tiempo para la preparación (93\%) y la discusión en grupos (87\%) es útil para el aprendizaje de los estudiantes. Sin embargo, un número significativo de docentes está de acuerdo o muy de acuerdo en que no conocen lo suficiente sobre las estrategias de enseñanza de las ciencias naturales basadas en la indagación (47\%). Además, sienten que no poseen el suficiente conocimiento de la materia en todos los temas $(47 \%)$ y alrededor del $36 \%$ no está satisfecho con su nivel actual de enseñanza. Estos resultados se pueden respaldar en Camargo et al. (2004), sobre las necesidades de formación permanente del docente los cuales en muchas ocasiones demuestran deficiencias disciplinares y pedagógicas.
Cerca del $67 \%$ están de acuerdo o muy de acuerdo en que utilizan la mayor parte del tiempo para presentar los contenidos (el docente habla), mientras que un poco más de la mitad de los docentes (53\%) no comparten sus resultados de aprendizaje con sus estudiantes. Igual de importante, más de la mitad de los docentes manifestaron que no tienen tiempo para trabajar con sus compañeros y que la mayoría de los docentes no tienen la oportunidad de asistir a capacitaciones y talleres para mejorar su enseñanza. Las anteriores preguntas son de preocupación ya que aparentemente los docentes de básica primaria en muchos casos carecen del suficiente conocimiento científico y confianza para enseñar ciencias (Ortiz \& Cervantes, 2016).

\section{FACTORES LIMITANTES Y FORMAS DE MEJORAR EL APRENDIZAJE Y LA ENSEÑANZA DE LA CIENCIAS NATURALES}

Tabla 8. Factores que limitan la enseñanza de las ciencias en las escuelas primarias.

\begin{tabular}{|l|c|}
\hline Factores & $\%$ \\
Falta de formación en la materia & 44.0 \\
Número de horas de enseñanza al día & 17.3 \\
Alto número de estudiantes por clase & 43.0 \\
Inasistencia de los estudiantes & 7.3 \\
Falta de material didáctico y su uso eficaz & 86.6 \\
Falta de espacio suficiente en las aulas & 42.0 \\
Menos información en el libro de texto & 6.6 \\
Enfoques de enseñanza-aprendizaje deficientes y bajo nivel de competencias & 12.0 \\
para enseñar & \\
Falta de conocimiento de la materia & 5.3 \\
Falta de tiempo para planificar y preparar las clases & 2.0 \\
\hline
\end{tabular}

Los datos de la tabla 8 muestran que la gran mayoría de los docentes incluidos en la muestra $(87 \%)$ indican que los factores más importantes que limitan la calidad del aprendizaje y la enseñanza de las ciencias naturales es la falta de material didáctico y su uso eficaz. Un número significativo de docentes informó de una falta de formación (44\%), la falta de espacio suficiente en las aulas (42\%) y el alto número de estudiantes por clase (43\%). Una minoría de docentes informó sobre sus muchas horas lectivas de clase, la falta de competencias para enseñar y el desconocer nuevos enfoques de enseñanza, que eran factores importantes que limitan la enseñanza de las ciencias naturales. 
Tabla 9. Sugerencias para mejorar el aprendizaje y la enseñanza.

\begin{tabular}{|l|l|c|c|}
\hline \multirow{5}{*}{ Sector } & Sugerencias de mejora & N & $\%$ \\
\hline \multirow{5}{*}{ Docentes } & $\begin{array}{l}\text { Desarrollo y mejora de sus competencias para enseñar } \\
\text { ciencias }\end{array}$ & 10 & 66.7 \\
\cline { 2 - 4 } & Formación continua y perfeccionamiento docente & 1 & 6.7 \\
\cline { 2 - 4 } & $\begin{array}{l}\text { Colaboración entre docentes como factor de aprendizaje y } \\
\text { promotor de mejora }\end{array}$ & 3 & 20.0 \\
\hline \multirow{5}{*}{ Materiales } & Uso adecuado de los recursos en el aula & 12 & 80.0 \\
\cline { 2 - 4 } & Aulas con espacio suficiente para enseñar ciencias & 5 & 33.3 \\
\cline { 2 - 4 } & Uso de recursos TIC & 2 & 13.3 \\
\cline { 2 - 4 } & Instalaciones de laboratorio & 1 & 6.7 \\
\cline { 2 - 4 } & Libros actualizados & 1 & 6.7 \\
\hline \multirow{5}{*}{ Pedagogía } & Utilizar la evaluación para el aprendizaje & 1 & 6.7 \\
\cline { 2 - 4 } & Proporcionar actividades prácticas y laboratorios & 5 & 33.3 \\
\cline { 2 - 4 } & Más intensidad horaria para las clases de ciencias & 1 & 6.7 \\
\cline { 2 - 4 } & Organizar la feria de las ciencias & 1 & 6.7 \\
\hline
\end{tabular}

Los datos que se muestran en la Tabla 9 muestran que, para mejorar la calidad de la enseñanza de las ciencias naturales, la mayoría de los

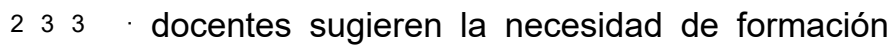
para desarrollar y mejorar sus competencias (65\%) y la dotación con mejores recursos $(80 \%)$.

Tabla 10. Opinión de los estudiantes para mejorar su aprendizaje de las ciencias.

\begin{tabular}{|l|c|c|}
\hline Sugerencias de mejora & N & $\%$ \\
El uso de materiales adecuados en el aula & 19 & 31.6 \\
Los docentes dan la oportunidad de que los estudiantes hablen en el aula & 6 & 10.0 \\
Los docentes dan instrucciones claras a los estudiantes & 8 & 13.3 \\
Los docentes tienen una actitud positiva ante la respuesta de los estudiantes & 8 & 13.3 \\
Los docentes dan deberes y los revisan regularmente & 7 & 11.6 \\
Los docentes hacen que los estudiantes participen en actividades prácticas. & 16 & 26.6 \\
Los docentes evalúan el aprendizaje de los estudiantes revisando los & 8 & 13.3 \\
cuadernos & &
\end{tabular}

Según la tabla 10, los estudiantes sugirieron que el estudio de las ciencias puede mejorarse mediante una mejor asignación de recursos; involucrar a los estudiantes con actividades prácticas; dar una enseñanza más clara y una actitud más solidaria con los problemas del estudiante. Un número similar afirmó que se necesita una mejor evaluación para apoyar su aprendizaje. Se evidenció que los estudiantes tenían poca o ninguna experiencia en técnicas de evaluación del aprendizaje y desconocían sus beneficios. 


\section{CONCLUSIÓN}

Los resultados muestran que, aunque el propósito de la enseñanza de la ciencia es desarrollar conocimientos científicos en los estudiantes, los docentes se centran más en las evaluaciones escritas. El proceso educativo de las ciencias naturales se centra en el docente, por lo tanto, su enseñanza es teórica. La evaluación se usa es para calificar y no para aprender. También hay una falta de recursos didácticos, espacio insuficiente en el aula, y los docentes están sobrecargados con sus clases. Para mejorar la calidad de la enseñanza, los docentes deben mejorar su formación en ciencias y estrategias pedagógicas basadas en la indagación. Se recomienda que los docentes se centren más en actividades prácticas que en enfoques teóricos.

\section{REFERENCIAS BIBLIOGRÁFICAS}

Aguilar-Barreto, A., Velandia-Riaño, Y. R., Aguilar-Barreto, C. P., \& Álvarez, G. R. (2017). Gestión educativa: tendencias de las políticas públicas educativas implementadas en Colombia. Revista Perspectivas, 2(2), 84-94. https://doi. org/10.22463/25909215.1331

Albarracín, C. Z., Hernández, C. A. \& Rojas, J. P. (2020). Objeto virtual de aprendizaje para desarrollar las habilidades numéricas: una experiencia con estudiantes de educación básica. Panorama, 14(26), 111-133. https://doi.org/10.15765/pnrm. v14i26.1486

Caldeira, M. H. (2005). Los libros de texto de ciencias: ¿son cómo deberían ser? Tarbiya, Revista De Investigación E Innovación Educativa, (36). https:// revistas.uam.es/tarbiya/article/ view/7240
Camargo, M., Calvo, G., Franco, M. C., Vergara, M., Londoño, S., Zapata, F. \& Garavito, C. (2004). Las necesidades de formación permanente del docente. Educación $y$ educadores, 7, 79-112. https:// educacionyeducadores.unisabana.edu. co/index.php/eye/article/view/550/643

Casas, J., Repullo, J. R. \& Donado, J. (2003). La encuesta como técnica de investigación. Elaboración de cuestionarios y tratamiento estadístico de los datos (I). Atención primaria, 31(8), 527538. https://doi.org/10.1016/S0212$\underline{6567(03) 79222-1}$

Echazarra, A. \& Schwabe, M. (2019). Colombia - Country Note - PISA 2018 Results. OECD. https://www.oecd.org/pisa/ publications/PISA2018_CN_COL_ESP. pdf

Fernández-Cezar, R., Adriano-Rincón, G., \& Prada-Núñez, R. (2019). ¿Se relacionan las creencias sobre las matemáticas con el rendimiento académico en matemáticas en estudiantes de contexto vulnerables? Eco matemático, 10(2), 6-15. http://dx.doi. org/10.22463/17948231.2588

Gamboa, A. A., Hernández, C. A. \& Prada, R. (2020). Competencias científicas, investigativas y comunicativas: experiencias desde una línea de investigación en enseñanza de las Ciencias naturales. Plumilla Educativa, 25(1), 13-26. https://doi.org/10.30554/ pe.1.3827.2020

Hernández, C. A., Gamboa, A. A. \& Prada R. (2021). Asociación entre memoria y rendimiento en matemáticas: un estudio correlacional. Revista Boletín Redipe, 10(4), 190-201. https://doi. org/10.36260/rbr.v10i4.1262 
Hernández, C. A., Gamboa, A. A. \& Prada, R. (2021). Competencias de maestros de ciencias naturales: una lectura desde las prácticas pedagógicas. Revista Boletín Redipe, 10(3), 360-375. https:// doi.org/10.36260/rbr.v10i3.1240

Hernández, C. \& Salamanca, X. (2018). Fortalecimiento de competencias científicas. Horizontes Pedagógicos, 19(2), 91-100. https://doi. org/10.33881/0123-8264.hop.19205

Hernández, C. A., Gamboa, A. A. \& Avendaño, W. R. (2020). Evaluación de carácter diagnóstico formativa en maestros de Ciencias naturales: análisis desde la reflexión y planeación de la práctica pedagógica. Revista Espacios, 41(33). 200-211. http://es.revistaespacios.com/ a20v41n33/a20v41n33p17.pdf

Hernández-Suárez, C., Avendaño-Castro, W. \& Rojas-Guevara, J. (2021). Planeación curricular y ambiente de aula en ciencias naturales: de las políticas y los lineamientos a la aplicación institucional. Revista de Investigación, Desarrollo e Innovación, 11(2), 319-334. https://doi.org/10.19053/20278306.v11. $\underline{\mathrm{n} 2.2021 .12758}$

Hernández-Suárez, C., Méndez-Umaña, J. P. \& Jaimes-Contreras, L. A. (2021). Memoria de trabajo y habilidades matemáticas en estudiantes de educación básica. Revista Científica, 40(1), 63-73. https:// doi.org/10.14483/23448350.15400

Hernández-Suárez, C., Pabón-Galán, C. \& Prada-Núñez, R. (2017). Desarrollo de competencias y su relación con el contexto educativo entre docentes de ciencias naturales. Revista Virtual Universidad Católica del Norte, 51, 194-215. http://revistavirtual.ucn.edu.
co/index.php/RevistaUCN/article/ view/852/1370

Ministerio de Educación Nacional. (1998). Lineamientos curriculares Ciencias Naturales y Educación Ambiental. Mineducación. https:// www.mineducacion.gov.co/1621/ articles89869_archivo_pdf5.pdf

Ministerio de Educación Nacional. (2004). Estándares Básicos de Competencias en Ciencias Naturales y Ciencias Sociales. Mineducación. https:// www.mineducacion.gov.co/1621/ articles81033_archivo_pdf.pdf

Ministerio de Educación Nacional. (2014). Todos a aprender: Programa para la Transformación de la Calidad Educativa. https://www.mineducacion.gov.co/1621/ articles-299245 recurso 1.pdf

Ministerio de Educación Nacional. (2016). Derechos básicos de aprendizaje Ciencias naturales V1. Mineducación. https://aprende.colombiaaprende.edu. co/sites/default/files/naspublic/DBA_C. Naturales.pdf

MontesA.(2017). Calidaddelaeducación primaria en Colombia: conceptualizaciones y tendencias. Escenarios, 15(2) 70-81. https://doi.org/10.15665/esc.v15i2.1624

Mora, B., \& Hernández, C. (2017). Las aulas invertidas: una estrategia para enseñar y otra forma de aprender física. INVENTUM, 12(22), 42-51. https://doi.org/10.26620/uniminuto. inventum.12.22.2017.42-51

Organización para la Cooperación y el Desarrollo Económicos. (2016). Pisa. Estudiantes de bajo rendimiento. Por qué se quedan atrás y cómo ayudarles a tener éxito. OCDE https://www.oecd.org/pisa/ 
keyfindings/PISA-2012-Estudiantes-debajo-rendimiento.pdf

Ortiz, D. (2015). El constructivismo como teoría y método de enseñanza. Sophia, (19), 93-110. https://doi.org/10.17163/soph. $\underline{\mathrm{n} 19.2015 .04}$

Ortiz, G., \& Cervantes, M. L. (2016). La formación científica en los primeros años de escolaridad. Panorama, 9(17), 10-23. https://doi.org/10.15765/pnrm.v9i17.788

Palma, K. (2017). Los principios didácticos constructivistas como prácticas inclusivas en el aula de primaria. Innovaciones Educativas, 19(27), 41-54. https://doi.org/10.22458/ie.v19i27.1954

Prada, R., Rincón, G. A. \& Hernández, C. A., (2018). Inteligencias múltiples y rendimiento académico del área de matemáticas en estudiantes de educación básica primaria. Infancias Imágenes, 17(2), 163-175. https://doi. org/10.14483/16579089.12584

Rincón, G. A., Hernández, C. A. \& Prada N, R. (2017). Influencia de los movimientos sacádicos en el rendimiento académico de estudiantes de básica primaria en situación de vulnerabilidad en la ciudad de Cúcuta. Psicogente, 20(38), 256-267. https://doi.org/10.17081/ psico.20.38.2545

Salamanca, X., \& Hernández Suárez, C. A. (2018). Enseñanza en ciencias naturales: la investigación como estrategia pedagógica. Trilogía Ciencia Tecnología Sociedad, 10(19), 133-148. https://doi.org/10.22430/21457778.1025

Tapia, M., Sánchez, C., Flores, V., Heeren, M., Jaramillo, D. \& Medina, A. (2011). Comprensión del fenómeno de la escucha en estudiantes de primer año medio de dos colegios particulares subvencionados de la comuna de Concepción. Enunciación, 16(1), 84-99. https://doi.org/10.14483/22486798.3591

Vargas, E. (2014). Prácticas evaluativas en la educación básica primaria en el Municipio de Pereira [tesis doctoral, Centro de Estudios Avanzados en Niñez y Juventud alianza de la Universidad de Manizales y el CINDE]. Repositorio Institucional CINDE. http://biblioteca. clacso.edu.ar/Colombia/alianza-cindeumz/20160518045859/EdilmaVargas. pdf

Veloza, R. A. \& Hernández, C. A. (2018a). Valoración de las estrategias adoptadas por docentes en la enseñanza de la ciencia desde la perspectiva de los estudiantes de educación básica. ÁNFORA, 25(45), 43-69. https://doi. org/10.30854/anf.v25.n45.2018.512

Veloza, R. A. \& Hernández, C. A. (2018b). The Assessment of the strategies adopted by teachers in the teaching of science from the perspective of basic education students. ÁNFORA, 25(45), 17-42. https://doi.org/10.30854/anf.v25. $\underline{\mathrm{n} 45.2018 .510}$

Zona-López, J. R. \& Giraldo-Márquez, J. D. (2017). Resolución de problemas: escenario del pensamiento crítico en la didáctica de las ciencias. Revista Latinoamericana de Estudios Educativos (Colombia), 13(2), 122-150. http:// dx.doi.org/10.17151/rlee.2017.13.2.8 